\title{
22. GRAIN SIZE OF SEDIMENTS FROM THE WESTERN EQUATORIAL PACIFIC: LEG 7, GLOMAR CHALLENGER
}

\author{
E. L. Gealy, Scripps Institution of Oceanography, \\ University of California, La Jolla, California
}

\begin{abstract}
METHODS
The grain size of sediments collected on Leg 7 was determined on shore at Scripps Institute of Oceanography by standard grain size analysis techniques (c.f., Krumbein and Pettijohn, 1938). Details of the actual procedures used are set forth in Volume IV of the Initial Reports of the Deep Sea Drilling Project (1970).

In general, from each core section recovered, one 10-gram sample of fresh sediment was taken in a plastic sampling tube. The samples were sealed in plastic vials and refrigerated until analyzed. In the laboratory, the samples were extruded into beakers, dried, and disaggregated in a dilute Calgon solution. The mixture was sieved through a 0.062 -millimeter sieve to remove the sand-sized particles, and the remainder was dispersed in a 1000-milliliter cylinder of water and pipetted to determine the silt and clay-sized fractions. It should be remembered that skeletal debris, Radiolaria skeletons in particular, have a high specific surface area, and their settling velocity is slower than their nominal diameter would indicate. Therefore, determination of the percentage of clay by pipette analysis may yield values that are too large in biogenous sediments.
\end{abstract}

The percentage of the total weight of the samples having the following equivalent diameters was determined:

$$
\begin{array}{ll}
\text { Sand: } & 0.062 \text { millimeter } \\
\text { Silt: } & 0.062 \text { to } 0.004 \text { millimeter } \\
\text { Clay: } & 0.004 \text { millimeter }
\end{array}
$$

A complete list of grain size results from all sites is given in Table 1. Size classifications shown in the table are after Shepard (1954). It should be emphasized that these classifications refer to particle size distribution only and in no way represent mineralogy. For example, in the ooze-chalk sequences, almost all of the sand fractions consist of foraminifera and Radiolaria tests. The clay fraction consists mostly of nannofossils and fragments of foraminifera tests. Triangular diagrams for each site are shown in Figure 1. The percentage of clay, silt and sand are shown plotted as a function of depth on both the core description charts and on the Site Summaries in Chapters 3 through 9.
Analyses of samples from lithified materials which are more difficult to disaggregate should be used with caution, as erroneously large proportions of either large-sized fractions or small-sized fractions may result from treatment. The samples analyzed may consist in part of particles which are aggregates of finer material, and analyses yield erroneously large proportions of the larger sizes. On the other hand, more heroic means are necessary to disperse partially lithified materials (the addition of hydrogen peroxide, agitation with an ultrasonic probe, etc.). This treatment can result in pulverizing some discrete particles, particularly more fragile ones, such as foraminifera and Radiolaria tests, and analyses of these materials will show an erroneously large proportion of finer material.

\section{RESULTS}

\section{Site 61}

Grain size analysis was made of only one sample of silty clay from Hole 61.1-1-2.

\section{Site 62}

Grain size analyses of samples from Site 62 indicate that the calcareous nannofossil ooze-chalk sequence penetrated increases irregularly (Quaternary) in grain size from a silty clay at the surface to a sand-silt-clay at 300 meters (Middle Miocene). Lower Miocene samples from Cores 4 and 5 are somewhat finer grained than the overlying material. Because few aggregates were present in the coarse fraction of the samples, the increase in grain size with depth and age appears to be real. There is some indication that zones of larger grain size may correspond to zones of higher porosity; both may relate to higher content of foraminifera tests.

\section{Site 63}

The grain size analyses of samples from Site 63 indicate that the nannofossil marl and chalk-ooze, with few exceptions, consists of silty clay sizes throughout, from Early Oligocene through Quaternary.

As in samples from Site 62, there appears to be a correlation between larger grain size and high porosity, and both may relate to content of foraminifera tests. 

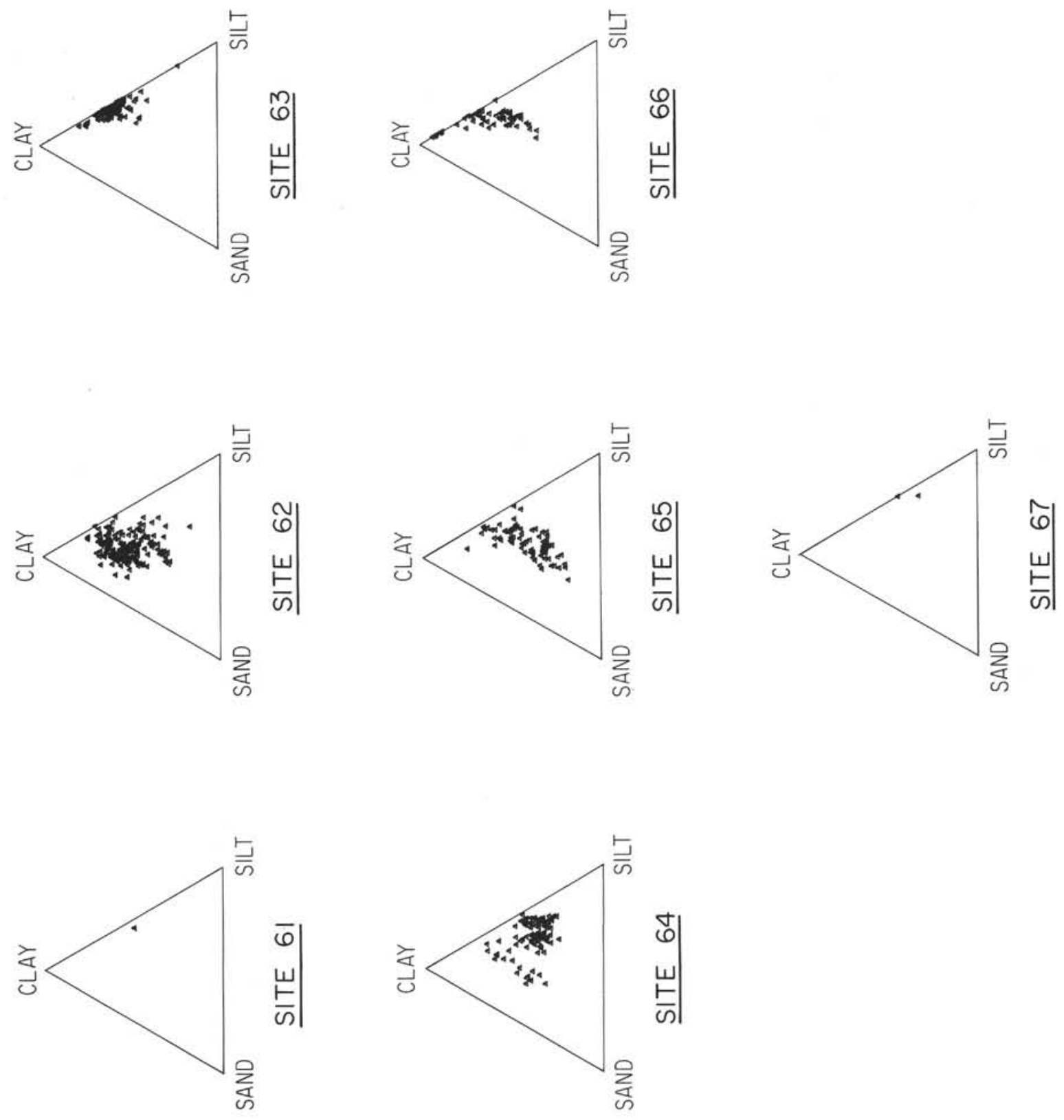

Figure 1. Triangle Diagrams of Grain Size: Leg 7 


\section{Site 64}

Grain size analyses of samples from Site 64 indicate that the nannofossil ooze-chalk sequence decreases in grain size with depth. There is a marked decrease in grain size from the Quaternary nannofossil ooze (35 per cent sand) near the sea floor to the Late Miocene chalk ooze (12 per cent sand) cored at 200 meters. The grain size of samples recovered between 200 and 600 meters (Late Oligocene) is relatively constant. The grain size decreases with depth in the interval between 600 meters and the Middle Eocene chalk (5 per cent sand) at 910 meters.

\section{Site 65}

The Middle Eocene-Quaternary sequence penetrated consists of radiolarian ooze throughout. A consistent increase in grain size with depth from near surface Quaternary oozes to the Late Eocene ooze-chalks at 120 meters is evident in both the proportion of sand-size fraction which increases from less than 10 per cent to 35 per cent, and the proportion of clay-size fraction which decreases from 60 per cent to 20 per cent. There is a slight decrease in grain size in samples between 120 meters and the Middle Eocene oozes at 160 meters ( 28 per cent sand sizes).

The fact that the increase in grain size with depth between Quaternary and Eocene material is indicated both by a decrease in the proportion of clay sizes and by an increase in the proportion of sand sizes (the latter being determined by sieving) suggests that the increase in clay sizes is largely real, and not an artifact introduced by inferring grain diameter from settling velocity. However, all of the values of the percentage of clay may be somewhat too high. (Size analysis by settling velocity of sediment containing a large proportion of skeletons, Radiolaria in particular, may yield erroneously high proportions of finer fractions.)

That the decrease in grain size from Eocene to Quaternary is not primarily due to increased fragmenting of skeletal material is suggested by the fact that the mean grain density of the ooze increases markedly between Eocene and Quaternary materials at Site 65. In Chapter 25, it is shown that the proportion of sand sizes increases and the proportion of clay sizes decreases as the measured mean grain density decreases in the Radiolarian ooze at Site 65 . This suggests that variations in grain size result primarily from variations in the content of opaline skeletal debris (grain density $\sim 2.0$ ), the balance consisting of finer, more dense components.

\section{Site 66}

As at Site 65, the Radiolarian ooze sequence shows an increase in grain size from Quaternary samples at 10 meters (less than 1 per cent sand sizes) through Early Miocene samples at 120 meters (22 per cent sand sizes). Like samples from Site 65, samples from this ooze also show a decrease in mean grain density with depth. This fact, together with the increase in grain size, suggests that the percentage of Radiolarian tests increases with depth which may relate to a decrease in productivity and/or an increase in bottom residence time from the Miocene through the Quaternary.

The pelagic clay (Cretaceous) from 165 to 190 meters is very fine-grained. Negligible sand sizes are reported, and the silt content is less than 15 per cent in 11 or 14 sections where samples were taken.

\section{Site 67}

Only two samples from Site 67 were analyzed for grain size. Both (67.0-1-1 and 67.1-1-2) were clayey silts and showed negligible sand content.

\section{REFERENCES}

Bader, R. D. et al., 1970. Appendix III. In Initial Reports of the Deep Sea Drilling Project, Volume $I V$. Washington D.C. (U.S. Government Printing Office).

Krumbein, W. C. and Pettijohn, F. J., 1938. Manual of Sedimentary Petrography. New York (AppletonCentury-Crofts, Inc.)

Shepard, F. P., 1954. Nomenclature based on sand-siltclay ratios. J. Sed. Petro. 24, 151. 
TABLE 1

Grainsize of Sediments from the Western Equatorial Pacific

\begin{tabular}{|c|c|c|c|c|c|c|c|c|c|c|c|}
\hline \multirow[b]{2}{*}{$\begin{array}{l}\text { Identifi- } \\
\text { cation }\end{array}$} & \multicolumn{5}{|c|}{ Grainsize $^{a}$} & \multirow[b]{2}{*}{$\begin{array}{l}\text { Identifi- } \\
\text { cation }\end{array}$} & \multicolumn{5}{|c|}{ Grainsize $^{\mathrm{a}}$} \\
\hline & $\begin{array}{c}\text { Interval } \\
\mathrm{cm}\end{array}$ & $\begin{array}{c}\text { Sand } \\
\text { Per Cent }\end{array}$ & $\begin{array}{c}\text { Silt } \\
\text { Per Cent }\end{array}$ & $\begin{array}{c}\text { Clay } \\
\text { Per Cent }\end{array}$ & Classification & & $\begin{array}{c}\text { Interval } \\
\mathrm{cm}\end{array}$ & $\begin{array}{c}\text { Sand } \\
\text { Per Cent }\end{array}$ & $\begin{array}{c}\text { Silt } \\
\text { Per Cent }\end{array}$ & $\begin{array}{c}\text { Clay } \\
\text { Per Cent }\end{array}$ & Classification \\
\hline Hole 61.1 & & & & & & Core 9-2 & 2.0 & 9.3 & 23.2 & 67.5 & Silty Clay \\
\hline Core $1-2$ & 42.0 & 4.3 & 45.9 & 49.8 & Silty Clay & $9-3$ & 2.0 & 19.1 & 19.2 & 61.7 & Silty Clay \\
\hline & & & & & & $9-4$ & 2.0 & 23.2 & 16.0 & 60.8 & Sandy Clay \\
\hline & & & & & & Core $10-1$ & 2.0 & 19.8 & 23.2 & 57.1 & Silty Clay \\
\hline Core 1-1 & 1.0 & 11.0 & 24.3 & 64.7 & Silty Clay & $10-2$ & 22.0 & 15.6 & 28.5 & 55.9 & Silty Clay \\
\hline Core 2-2 & 3.0 & 3.6 & 32.4 & 64.1 & Silty Clay & $10-3$ & 10.0 & 24.7 & 22.4 & 52.9 & Sand-Silt-Clay \\
\hline $2-3$ & 8.0 & 6.3 & 33.0 & 60.8 & Silty Clay & $10-4$ & 5.0 & 22.6 & 21.0 & 56.4 & Sand-Silt-Clay \\
\hline $2-4$ & 3.0 & 4.2 & 33.7 & 62.1 & Silty Clay & $10-5$ & 2.0 & 13.6 & 19.0 & 67.4 & Silty Clay \\
\hline Core $3-1$ & 2.0 & 14.6 & 40.1 & 45.3 & Silty Clay & $10-6$ & 3.0 & 26.5 & 21.8 & 51.6 & Sand-Silt-Clay \\
\hline $3-2$ & 2.0 & 11.2 & 37.9 & 50.9 & Silty Clay & Core $11-2$ & 2.0 & 12.6 & 26.0 & 61.4 & Silty Clay \\
\hline $3-3$ & 2.0 & 11.4 & 43.5 & 45.1 & Silty Clay & $11-4$ & 3.0 & 17.8 & 20.3 & 61.9 & Silty Clay \\
\hline $3-4$ & 2.0 & 15.8 & 40.7 & 43.4 & Silty Clay & $11-5$ & 2.0 & 20.6 & 33.1 & 46.3 & Sand-Silt-Clay \\
\hline $3-5$ & 2.0 & 14.3 & 40.2 & 45.5 & Silty Clay & $11-6$ & 28.0 & 12.0 & 24.8 & 63.2 & Silty Clay \\
\hline $3-6$ & 2.0 & 13.1 & 40.5 & 46.4 & Silty Clay & Core $12-1$ & 2.0 & 18.9 & 25.8 & 55.3 & Silty Clay \\
\hline Core 4-5 & 2.0 & 13.6 & 51.7 & 34.7 & Clayey Silt & $12-2$ & 2.0 & 19.2 & 24.4 & 56.4 & Silty Clay \\
\hline Core $5-2$ & 14.0 & 14.2 & 47.6 & 38.2 & Clayey Silt & $12-3$ & 5.0 & 29.3 & 11.7 & 59.0 & Sandy Clay \\
\hline $5-3$ & 1.0 & 9.4 & 47.5 & 43.1 & Clayey Silt & $12-4$ & 2.0 & 25.8 & 24.8 & 49.4 & Sand-Silt-Clay \\
\hline & & & & & & $12-5$ & 2.0 & 11.2 & 23.6 & 65.2 & Silty Clay \\
\hline Hole 62.1 & & & & & & $12-6$ & 2.0 & 22.1 & 19.8 & 58.1 & Sandy Clay \\
\hline Core $1-2$ & 30.0 & 2.4 & 29.3 & 68.3 & Silty Clay & Core $13-3$ & 91.0 & 7.9 & 24.9 & 67.3 & Silty Clay \\
\hline $1-3$ & 4.0 & 2.9 & 36.6 & 60.4 & Silty Clay & $13-4$ & 5.0 & 16.4 & 23.1 & 60.4 & Silty Clay \\
\hline Core 4-1 & 2.0 & 16.5 & 28.9 & 54.7 & Silty Clay & $13-5$ & 2.0 & 22.0 & 21.9 & 56.0 & Sand-Silt-Clay \\
\hline $4-3$ & 2.0 & 6.8 & 30.9 & 62.3 & Silty Clay & $13-6$ & 4.0 & 21.6 & 22.5 & 55.9 & Sand-Silt-Clay \\
\hline $4-4$ & 2.0 & 5.0 & 25.5 & 69.5 & Silty Clay & Core $14-1$ & 51.0 & 26.8 & 21.4 & 51.9 & Sand-Silt-Clay \\
\hline $4-5$ & 4.0 & 4.3 & 32.7 & 63.0 & Silty Clay & $14-2$ & 5.0 & 28.1 & 20.0 & 51.9 & Sand-Silt-Clay \\
\hline $4-6$ & 2.0 & 12.0 & 33.3 & 54.8 & Silty Clay & $14-3$ & 2.0 & 30.8 & 18.3 & 50.9 & Sandy Clay \\
\hline Core 6-1 & 10.0 & 5.3 & 26.9 & 67.7 & Silty Clay & $14-4$ & 5.0 & 24.6 & 22.8 & 52.6 & Sand-Silt-Clay \\
\hline $6-2$ & 3.0 & 11.0 & 26.8 & 62.2 & Silty Clay & $14-5$ & 6.0 & 39.0 & 32.0 & 29.0 & Sand-Silt-Clay \\
\hline $6-3$ & 2.0 & 8.3 & 22.9 & 68.8 & Silty Clay & $14-6$ & 12.0 & 22.6 & 21.3 & 56.1 & Sand-Silt-Clay \\
\hline $6-4$ & 2.0 & 7.4 & 28.1 & 64.4 & Silty Clay & Core 15-1 & 2.0 & 31.5 & 22.5 & 45.9 & Sand-Silt-Clay \\
\hline $6-5$ & 2.0 & 10.0 & 24.1 & 65.9 & Silty Clay & & & & & 672 & Silty Clay \\
\hline $6-6$ & 2.0 & 33.5 & 14.0 & 52.4 & Sandy Clay & Core 16-1 & 3.0 & 8.7 & $\begin{array}{l}24.1 \\
22.5\end{array}$ & $\begin{array}{l}67.2 \\
74.3\end{array}$ & $\begin{array}{l}\text { Silty Clay } \\
\text { Silty Clay }\end{array}$ \\
\hline Core $7-1$ & 4.0 & 4.1 & 21.3 & 74.6 & Silty Clay & $\begin{array}{l}16-3 \\
16-4\end{array}$ & $\begin{array}{l}4.0 \\
2.0\end{array}$ & $\begin{array}{r}3.2 \\
23.5\end{array}$ & $\begin{array}{l}22.5 \\
21.4\end{array}$ & $\begin{array}{l}14.3 \\
55.0\end{array}$ & Sand-Silt-Clay \\
\hline $7-2$ & 2.0 & 20.3 & 19.4 & 60.3 & Sandy Clay & $16-6$ & 3.0 & 14.5 & 23.3 & 62.2 & Silty Clay \\
\hline $7-3$ & 2.0 & 9.7 & 27.5 & 62.8 & Silty Clay & & & & & & \\
\hline $7-4$ & 2.0 & 19.2 & 20.3 & 60.5 & Silty Clay & Core 17-1 & 122.0 & 15.7 & 16.2 & 68.1 & Silty Clay \\
\hline $7-5$ & 2.0 & 21.7 & 21.0 & 57.3 & Sand-Silt-Clay & $17-2$ & 6.0 & 17.3 & 17.5 & 65.2 & Silty Clay \\
\hline $7-6$ & 2.0 & 3.5 & 21.6 & 74.8 & Silty Clay & $17-4$ & 4.0 & 10.8 & 22.5 & 66.7 & Silty Clay \\
\hline Core 8-3 & 2.0 & 10.0 & 22.9 & 67.1 & Silty Clay & $17-6$ & 2.0 & 20.9 & 33.5 & 45.6 & Sand-Silt-Clay \\
\hline $8-4$ & 2.0 & 13.2 & 23.8 & 63.0 & Silty Clay & Core $18-1$ & 2.0 & 19.1 & 22.8 & 58.2 & Silty Clay \\
\hline $8-5$ & 2.0 & 13.5 & 17.0 & 69.6 & Silty Clay & 18.3 & 2.0 & 19.8 & 20.2 & 60.0 & Silty Clay \\
\hline $8-6$ & 2.0 & 11.6 & 26.2 & 62.2 & Silty Clay & $18-4$ & 2.0 & 20.7 & 27.3 & 51.9 & Sand-Silt-Clay \\
\hline
\end{tabular}

${ }^{\mathrm{a}}$ Grainsize: Sand per cent of total weight greater than .062 millimeter; clay per cent of total weight less than .0039 millimeter; silt remainder of total weight. 
TABLE 1 - Continued

\begin{tabular}{|c|c|c|c|c|c|c|c|c|c|c|c|}
\hline & & & Grainsize & & & & & & Grainsize & & \\
\hline $\begin{array}{l}\text { Identifi- } \\
\text { cation }\end{array}$ & $\begin{array}{c}\text { Interval } \\
\mathrm{cm}\end{array}$ & $\begin{array}{c}\text { Sand } \\
\text { Per Cent }\end{array}$ & $\begin{array}{c}\text { Silt } \\
\text { Per Cent }\end{array}$ & $\begin{array}{c}\text { Clay } \\
\text { Per Cent }\end{array}$ & Classification & $\begin{array}{l}\text { Identifi- } \\
\text { cation }\end{array}$ & $\begin{array}{c}\text { Interval } \\
\mathrm{cm}\end{array}$ & $\begin{array}{c}\text { Sand } \\
\text { Per Cent }\end{array}$ & $\begin{array}{c}\text { Silt } \\
\text { Per Cent }\end{array}$ & $\begin{array}{c}\text { Clay } \\
\text { Per Cent }\end{array}$ & Classification \\
\hline Hole 62-1 & - Continu & & & & & $25-4$ & 2.0 & 10.0 & 30.8 & 59.3 & Silty Clay \\
\hline $18-5$ & 4.0 & 21.6 & 27.0 & 51.4 & Sand-Silt-Clay & $25-5$ & 6.0 & 20.2 & 25.1 & 54.7 & Sand-Silt-Clay \\
\hline $18-6$ & 3.0 & 13.7 & 34.9 & 51.4 & Silty Clay & $25-6$ & 2.0 & 10.1 & 34.2 & 55.7 & Silty Clay \\
\hline Core 19-1 & 78.0 & 16.9 & 28.9 & 54.2 & Silty Clay & Core 26-1 & 7.0 & 20.1 & 26.6 & 53.3 & Sand-Silt-Clay \\
\hline $19-2$ & 30.0 & 26.1 & 25.3 & 48.6 & Sand-Silt-Clay & $26-2$ & 6.0 & 24.1 & 28.4 & 47.5 & Sand-Silt-Clay \\
\hline $19-3$ & 3.0 & 26.4 & 22.7 & 50.9 & Sand-Silt-Clay & $26-3$ & 3.0 & 18.2 & 27.0 & 54.8 & Silty Clay \\
\hline $19-4$ & 3.0 & 23.6 & 23.8 & 52.5 & Sand-Silt-Clay & $26-4$ & 3.0 & 20.5 & 25.0 & 54.5 & Sand-Silt-Clay \\
\hline $19-5$ & 2.0 & 25.8 & 25.9 & 48.4 & Sand-Silt-Clay & $26-6$ & 3.0 & 19.3 & 28.5 & 52.2 & Silty Clay \\
\hline $19-6$ & 3.0 & 18.8 & 27.8 & 53.4 & Silty Clay & Core 27-1 & 67.0 & 18.1 & 25.4 & 56.4 & Silty Clay \\
\hline Core $20-1$ & 4.0 & 31.8 & 24.1 & 44.1 & Sand-Silt-Clay & $27-2$ & 7.0 & 23.6 & 28.8 & 47.6 & Sand-Silt-Clay \\
\hline $20-2$ & 2.0 & 18.7 & 28.4 & 52.9 & Silty Clay & $27-3$ & 2.0 & 21.1 & 29.1 & 49.7 & Sand-Silt-Clay \\
\hline $20-3$ & 2.0 & 29.8 & 23.5 & 46.7 & Sand-Silt-Clay & $27-4$ & 2.0 & 26.5 & 26.3 & 47.2 & Sand-Silt-Clay \\
\hline $20-4$ & 2.0 & 20.4 & 25.6 & 54.0 & Sand-Silt-Clay & $27-5$ & 3.0 & 21.2 & 27.9 & 50.9 & Sand-Silt-Clay \\
\hline $20-5$ & 3.0 & 34.3 & 24.8 & 41.0 & Sand-Silt-Clay & Core 28-1 & 3.0 & 18.9 & 30.3 & 50.8 & Silty Clay \\
\hline $20-6$ & 4.0 & 13.1 & 29.9 & 57.0 & Silty-Clay & $28-2$ & 3.0 & 18.2 & 31.5 & 50.3 & Silty Clay \\
\hline Core $21-1$ & 4.0 & 28.5 & 24.1 & 47.4 & Sand-Silt-Clay & $28-3$ & 2.0 & 1.2 & 39.9 & 58.9 & Silty Clay \\
\hline $21-2$ & 11.0 & 31.9 & 24.9 & 43.2 & Sand-Silt-Clay & $28-4$ & 3.0 & 22.4 & 30.9 & 46.6 & Sand-Silt-Clay \\
\hline $21-3$ & 2.0 & 24.9 & 24.5 & 50.6 & Sand-Silt-Clay & $28-5$ & 2.0 & 28.5 & 30.9 & 40.6 & Sand-Silt-Clay \\
\hline $21-4$ & 3.0 & 24.8 & 20.7 & 54.5 & Sand-Silt-Clay & & & & & & \\
\hline $21-5$ & 3.0 & 22.6 & 24.5 & 52.9 & Sand-Silt-Clay & Core 29-1 & 3.0 & 24.9 & 32.7 & 42.4 & Sand-Silt-Clay \\
\hline $21-6$ & 3.0 & 21.7 & 20.8 & 57.5 & Sand-Silt-Clay & $29-2$ & 5.0 & 18.3 & 33.0 & 48.7 & Silty Clay \\
\hline Core $22-1$ & 3.0 & 15.7 & 20.2 & 64.2 & Silty Clay & $29-3$ & 2.0 & 24.8 & 28.6 & 46.6 & Sand-Silt-Clay \\
\hline $22-2$ & 2.0 & 21.1 & 20.7 & 583 & Sand-Silts & $29-4$ & 2.0 & 27.0 & 56.1 & 16.9 & Sandy Silt \\
\hline $22-3$ & 2.0 & 17.0 & 22.9 & & & $29-5$ & 2.0 & 19.9 & 49.5 & 30.6 & Clayey Silt \\
\hline $22-4$ & 3.0 & 20.2 & 19.1 & 00.1 & Silty Ciay & $29-6$ & 3.0 & 21.0 & 31.0 & 48.0 & Sand-Silt-Clay \\
\hline $22-5$ & 3.0 & 30.7 & 24.2 & 00.1 & Sandy Clay & & & & & & \\
\hline $22-6$ & 4.0 & 11.8 & $\begin{array}{l}24.2 \\
23.3\end{array}$ & 45.1 & Sand-Silt-Clay & Core $30-1$ & 2.0 & 25.9 & 27.7 & 46.5 & Sand-Silt-Clay \\
\hline & & & & 64.9 & Silty Clay & $30-2$ & 2.0 & 15.6 & 28.4 & 56.0 & Silty Clay \\
\hline $\begin{array}{r}23-1 \\
23-2\end{array}$ & 1.0 & 8.1 & 29.1 & 62.8 & Silty Clay & $30-3$ & 2.0 & 23.7 & 29.6 & 46.7 & Sand-Silt-Clay \\
\hline $23-2$ & 4.0 & 14.2 & 21.9 & 63.9 & Silty Clay & $30-4$ & 2.0 & 18.5 & 27.2 & 54.4 & Silty Clay \\
\hline $23-3$ & 2.0 & 19.1 & 20.6 & 60.2 & Silty Clay & $30-5$ & 2.0 & 22.3 & 27.7 & 50.0 & Sand-Silt-Clay \\
\hline $23-4$ & 3.0 & 14.2 & 22.6 & 63.2 & Silty Clay & $30-6$ & 2.0 & 9.8 & 33.2 & 57.0 & Silty Clay \\
\hline $23-5$ & 6.0 & 23.5 & 18.9 & 57.6 & Sandy Clay & Core 31-1 & 1.0 & 19.8 & 31.4 & 48.8 & Silty Clay \\
\hline $23-6$ & 3.0 & 10.3 & 25.4 & 64.3 & Silty Clay & $31-2$ & 2.0 & 16.4 & 36.0 & 47.6 & Silty Clay \\
\hline Core 24-1 & 3.0 & 16.6 & 18.4 & 65.0 & Silty Clay & $31-3$ & 4.0 & 20.5 & 33.9 & 45.6 & Sand-Silt-Clay \\
\hline $24-2$ & 13.0 & 22.7 & 19.8 & 57.5 & Sandy Clay & $31-4$ & 1.0 & 13.6 & 37.8 & 48.6 & Silty Clay \\
\hline $24-3$ & 2.0 & 0.0 & 29.6 & 70.4 & Silty Clay & $31-6$ & 1.0 & 13.1 & 44.0 & 42.9 & Clayey Silt \\
\hline $24-4$ & 3.0 & 18.8 & 20.7 & 60.5 & Silty Clay & Core $32-1$ & 1.0 & 23.6 & 37.5 & 39.0 & Sand-Silt-Clay \\
\hline $24-5$ & 16.0 & 26.4 & 17.7 & 55.9 & Sandy Clay & $32-2$ & 2.0 & 25.3 & 37.4 & 37.2 & Sand-Silt-Clay \\
\hline $24-6$ & 3.0 & 5.0 & 27.0 & 68.0 & Silty Clay & $32-3$ & 1.0 & 31.6 & 37.1 & 31.4 & Sand-Silt-Clay \\
\hline Core $25-1$ & 6.0 & 11.5 & 24.5 & 63.9 & Silty Clay & $32-4$ & 1.0 & 36.9 & 32.3 & 30.8 & Sand-Silt-Clay \\
\hline $25-2$ & 6.0 & 11.7 & 29.4 & 58.9 & Silty Clay & $32-5$ & 2.0 & 28.8 & 36.1 & 35.0 & Sand-Silt-Clay \\
\hline $25-3$ & 3.0 & 15.5 & 28.6 & 55.8 & Silty Clay & $32-6$ & 1.0 & 35.1 & 34.4 & 30.5 & Sand-Silt-Clay \\
\hline
\end{tabular}

${ }^{\mathrm{a}}$ Grainsize: Sand per cent of total weight greater than .062 millimeter; clay per cent of total weight less than .0039 millimeter, silt remainder of total weight. 
TABLE 1 - Continued

\begin{tabular}{|c|c|c|c|c|c|c|c|c|c|c|c|}
\hline & & & Grainsize & & & & & & Grainsize & & \\
\hline $\begin{array}{l}\text { Identifi- } \\
\text { cation }\end{array}$ & $\begin{array}{c}\text { Interval } \\
\mathrm{cm}\end{array}$ & $\begin{array}{c}\text { Sand } \\
\text { Per Cent }\end{array}$ & $\begin{array}{c}\text { Silt } \\
\text { Per Cent }\end{array}$ & $\begin{array}{c}\text { Clay } \\
\text { Per Cent }\end{array}$ & Classification & $\begin{array}{l}\text { Identifi- } \\
\text { cation }\end{array}$ & $\begin{array}{c}\text { Interval } \\
\mathrm{cm}\end{array}$ & $\begin{array}{c}\text { Sand } \\
\text { Per Cent }\end{array}$ & $\begin{array}{c}\text { Silt } \\
\text { Per Cent }\end{array}$ & $\begin{array}{c}\text { Clay } \\
\text { Per Cen }\end{array}$ & Classification \\
\hline Hole 62-1 & - Conti & tinued & & & & Core $7-1$ & 1.0 & 5.0 & 35.8 & 59.2 & Silty Clay \\
\hline & & & & & & $7-2$ & 2.0 & 3.8 & 39.4 & 56.8 & Silty Clay \\
\hline Core 33-2 & 0.0 & 32.9 & 35.1 & 32.0 & Sand-Silt-Clay & $7-3$ & 1.0 & 4.5 & 38.3 & 57.2 & Silty Clay \\
\hline $33-3$ & 1.0 & 25.9 & 35.7 & 38.4 & Sand-Silt-Clay & $7-4$ & 3.0 & 2.5 & 35.7 & 61.8 & Silty Clay \\
\hline Core $34-1$ & 4.0 & 19.4 & 36.3 & 44.3 & Silty Clay & $7-5$ & 1.0 & 3.2 & 39.3 & 57.5 & Silty Clay \\
\hline $34-4$ & 2.0 & 19.6 & 38.8 & 41.6 & Silty Clay & $7-6$ & 4.0 & 3.6 & 36.8 & 59.6 & Silty Clay \\
\hline $34-5$ & 4.0 & 26.3 & 38.7 & 35.0 & Sand-Silt-Clay & Core $8-1$ & 50.0 & 14.7 & 41.5 & 43.8 & Silty Clay \\
\hline Core $35-1$ & 30.0 & 18.7 & 38.4 & 42.9 & Silty Clay & $8-2$ & 3.0 & 4.9 & 40.3 & 54.8 & Silty Clay \\
\hline $35-2$ & 2.0 & 21.1 & 41.7 & 37.2 & Sand-Silt-Clay & $8-3$ & 2.0 & 5.0 & 39.7 & 55.3 & Silty Clay \\
\hline $35-3$ & 2.0 & 20.2 & 39.3 & 40.5 & Sand-Silt-Clay & Core 9-1 & 18.0 & 4.5 & 50.2 & 45.3 & Clayey Silt \\
\hline $35-4$ & 2.0 & 23.4 & 38.0 & 38.6 & Sand-Silt-Clay & 9-3 & 0.0 & 0.3 & 77.7 & 21.9 & Silt \\
\hline $35-5$ & 4.0 & 24.6 & 43.9 & 31.5 & Sand-Silt-Clay & $9-4$ & 0.0 & 3.0 & 34.0 & 63.1 & Silty Clay \\
\hline $35-6$ & 2.0 & 38.1 & 33.6 & 28.3 & Sand-Silt-Clay & Hole 63.1 & & & & & \\
\hline Core $36-2$ & 2.0 & 33.2 & 37.2 & 29.6 & Sand-Silt-Clay & & & & & & \\
\hline $36-3$ & 2.0 & 24.0 & 36.7 & 39.4 & Sand-Silt-Clay & Core 1-1 & 46.0 & 6.8 & 34.1 & 59.1 & Silty Clay \\
\hline $36-4$ & 2.0 & 20.2 & 12.5 & 67.3 & Sandy Clay & $1-2$ & 2.0 & 3.8 & 29.3 & 66.9 & Silty Clay \\
\hline $36-5$ & 2.0 & 31.6 & 34.5 & 33.9 & Sand-Silt-Clay & $1-3$ & 1.0 & 3.2 & 42.4 & 54.4 & Silty Clay \\
\hline Core $37-2$ & 2.0 & 30.8 & 35.9 & 33.3 & Sand-Silt-Clay & Core $3-1$ & 55.0 & 3.4 & 34.8 & 61.8 & Silty Clay \\
\hline & & & & & & $3-2$ & 1.0 & 9.6 & 35.8 & 54.6 & Silty Clay \\
\hline Hole 63.0 & & & & & & Core 5-3 & 1.0 & 0.2 & 35.9 & 64.0 & Silty Clay \\
\hline Core 1-1 & 3.0 & 3.7 & 22.8 & 73.5 & Silty Clay & $5-4$ & 3.0 & 0.5 & 33.5 & 66.0 & Silty Clay \\
\hline $1-2$ & 7.0 & 0.7 & 38.4 & 60.8 & Silty Clay & & & & & & \\
\hline $4,1-4$ & 2.0 & 1.5 & 36.6 & 61.9 & Silty Clay & Core 6-1 & 61.0 & 0.6 & 35.1 & 64.3 & Silty Clay \\
\hline $1-5$ & 10.0 & 5.3 & 48.4 & 46.3 & Clayey Silt & $6-2$ & 1.0 & 1.3 & 37.3 & 61.4 & Silty Clay \\
\hline $1-6$ & 4.0 & 4.3 & 40.4 & 55.3 & Silty Clay & $6-3$ & 4.0 & 0.9 & 39.3 & 59.8 & Silty Clay \\
\hline Core 2-2 & 2.0 & 1.8 & 20.7 & 77.5 & Clay & $6-4$ & 3.0 & 2.9 & 44.5 & 52.7 & Silty Clay \\
\hline $2-6$ & 1.0 & 2.6 & 23.7 & 73.7 & Silty Clay & $6-5$ & 3.0 & 0.6 & 31.7 & 67.7 & Silty Clay \\
\hline & & 2.6 & 34.1 & 63.3 & & $6-6$ & 1.0 & 0.9 & 30.5 & 68.6 & Silty Clay \\
\hline $\begin{array}{r}\text { Core } 3-1 \\
3-2\end{array}$ & 56.0 & $\begin{array}{l}2.6 \\
1.7\end{array}$ & $\begin{array}{l}34.1 \\
30.7\end{array}$ & $\begin{array}{l}63.3 \\
67.6\end{array}$ & Silty Clay & Core $7-2$ & 1.0 & 2.0 & 45.7 & 52.3 & Silty Clay \\
\hline $\begin{array}{l}3-2 \\
3-3\end{array}$ & $\begin{array}{l}4.0 \\
2.0\end{array}$ & $\begin{array}{l}1.1 \\
4.8\end{array}$ & 40.6 & $\begin{array}{l}67.6 \\
54.6\end{array}$ & $\begin{array}{l}\text { Silty Clay } \\
\text { Silty Clay }\end{array}$ & $7-3$ & 1.0 & 4.5 & 37.0 & 58.5 & Silty Clay \\
\hline $3-4$ & 1.0 & 2.0 & 36.2 & 61.8 & Silty Clay & & & & & & \\
\hline & & & & & & Core 8-2 & 10.0 & 4.3 & 34.8 & 60.9 & Silty Clay \\
\hline Core 4-1 & 7.0 & 1.0 & 36.7 & 62.3 & Silty Clay & $8-3$ & 13.0 & 2.1 & 40.8 & 57.1 & Silty Clay \\
\hline $4-2$ & 1.0 & 0.3 & 43.4 & 56.3 & Silty Clay & $8-4$ & 1.0 & 3.9 & 38.0 & 58.1 & Silty Clay \\
\hline Core $5-1$ & 14.0 & 2.6 & 43.6 & 53.8 & Silty Clay & $8-5$ & 2.0 & 1.6 & 42.7 & 55.7 & Silty Clay \\
\hline $5-2$ & 4.0 & 5.1 & 37.2 & 57.7 & Silty Clay & $8-6$ & 1.0 & 1.5 & 45.9 & 52.6 & Silty Clay \\
\hline Core 6-1 & 2.0 & 16.4 & 38.3 & 45.3 & Silty Clay & Core 9-1 & 10.0 & 1.0 & 36.6 & 62.4 & Silty Clay \\
\hline $6-2$ & 2.0 & 10.2 & 38.4 & 51.5 & Silty Clay & $9-2$ & 2.0 & 1.1 & 39.4 & 59.5 & Silty Clay \\
\hline $6-3$ & 2.0 & 6.4 & 31.8 & 61.8 & Silty Clay & $9-3$ & 2.0 & 1.7 & 39.0 & 59.3 & Silty Clay \\
\hline $6-4$ & 2.0 & 6.2 & 33.1 & 60.8 & Silty Clay & $9-4$ & 6.0 & 2.1 & 42.2 & 55.6 & Silty Clay \\
\hline $6-5$ & 2.0 & 7.2 & 37.3 & 55.5 & Silty Clay & $9-5$ & 2.0 & 1.0 & 40.4 & 58.5 & Silty Clay \\
\hline $6-6$ & 1.0 & 6.3 & 32.8 & 60.9 & Silty Clay & $9-6$ & 2.0 & 8.5 & 37.5 & 54.0 & Silty Clay \\
\hline
\end{tabular}

${ }^{\mathrm{a}}$ Grainsize: Sand per cent of total weight greater than .062 millimeter; clay per cent of total weight less than .0039 millimeter; silt remainder of total weight. 
TABLE 1 - Continued

\begin{tabular}{|c|c|c|c|c|c|c|c|c|c|c|c|}
\hline & & & Grainsize & & & & & & Grainsize & & \\
\hline $\begin{array}{l}\text { Identifi- } \\
\text { cation }\end{array}$ & $\begin{array}{l}\text { Interval } \\
\mathrm{cm}\end{array}$ & $\begin{array}{c}\text { Sand } \\
\text { Per Cent }\end{array}$ & $\begin{array}{c}\text { Silt } \\
\text { Per Cent }\end{array}$ & $\begin{array}{c}\text { Clay } \\
\text { Per Cent }\end{array}$ & Classification & $\begin{array}{l}\text { Identifi- } \\
\text { cation }\end{array}$ & $\begin{array}{c}\text { Interval } \\
\mathrm{cm}\end{array}$ & $\begin{array}{c}\text { Sand } \\
\text { Per Cent }\end{array}$ & $\begin{array}{c}\text { Silt } \\
\text { Per Cent }\end{array}$ & $\begin{array}{c}\text { Clay } \\
\text { Per Cent }\end{array}$ & Classification \\
\hline Hole 63.1 & - Conti & inued & & & & $1-5$ & 2.0 & 31.3 & 23.7 & 43.2 & Sand-Silt-Clay \\
\hline Core 10-1 & 10.0 & 0.5 & 45.2 & 54.2 & Silty Clay & $1-6$ & 73.0 & 35.7 & 23.7 & 40.6 & Sand-Silt-Clay \\
\hline $10-2$ & 2.0 & 1.5 & 45.4 & 53.2 & Silty Clay & Core 2-2 & 4.0 & 31.6 & 28.6 & 39.8 & Sand-Silt-Clay \\
\hline $10-5$ & 4.0 & 2.5 & 55.1 & 42.5 & Clayey Silt & $2-3$ & 5.0 & 20.7 & 28.3 & 51.1 & Sand-Silt-Clay \\
\hline Core 11-1 & 1.0 & 5.3 & 35.2 & 59.5 & Silty Clay & $2-4$ & 4.0 & 26.2 & 26.8 & 47.0 & Sand-Silt-Clay \\
\hline $11-2$ & 1.0 & 1.3 & 41.9 & 56.8 & Silty Clay & $2-6$ & 1.0 & 28.0 & 23.5 & 48.5 & Sand-Silt-Clay \\
\hline $11-3$ & 1.0 & 1.7 & 46.2 & 52.1 & Silty Clay & Core 3-1 & 1.0 & 13.6 & 26.8 & 59.6 & Silty Clay \\
\hline $11-4$ & 7.0 & 7.1 & 43.6 & 49.3 & Silty Clay & 3-2 & 4.0 & 17.4 & 26.6 & 56.0 & Silty Clay \\
\hline $11-5$ & 1.0 & 6.4 & 43.2 & 50.4 & Silty Clay & $3-3$ & 4.0 & 9.0 & 26.0 & 65.0 & Silty Clay \\
\hline $11-6$ & 1.0 & 1.1 & 39.8 & 59.1 & Silty Clay & $3-4$ & 5.0 & 13.3 & 26.5 & 60.1 & Silty Clay \\
\hline Core 13-1 & 1.0 & 0.5 & 36.6 & 62.9 & Silty Clay & $3-5$ & 2.0 & 14.9 & 24.1 & 61.0 & Silty Clay \\
\hline $13-2$ & 2.0 & 3.4 & 43.6 & 53.0 & Silty Clay & $3-6$ & 4.0 & 5.5 & 29.2 & 65.3 & Silty Clay \\
\hline $13-3$ & 1.0 & 5.0 & 39.1 & 55.9 & Silty Clay & Core 4-1 & 47.0 & 16.8 & 46.8 & 36.4 & Clayey Silt \\
\hline $13-4$ & 2.0 & 1.2 & 43.5 & 55.4 & Silty Clay & 4-2 & 8.0 & 15.5 & 47.6 & 36.9 & Clayey Silt \\
\hline $13-5$ & 8.0 & 5.4 & 37.7 & 56.9 & Silty Clay & 4-3 & 8.0 & 16.6 & 49.1 & 34.3 & Clayey Silt \\
\hline $13-6$ & 3.0 & 8.5 & 43.2 & 48.3 & Silty Clay & $4-4$ & 12.0 & 14.5 & 49.3 & 36.2 & Clayey Silt \\
\hline Core 14-1 & 2.0 & 1.9 & 37.4 & 60.7 & Silty Clay & $4-5$ & 21.0 & 14.4 & 54.0 & 31.6 & Clayey Silt \\
\hline $14-2$ & 1.0 & 0.8 & 46.2 & 53.0 & Silty Clay & $4-6$ & 4.0 & 17.4 & 42.4 & 40.1 & Clayey Silt \\
\hline $14-3$ & 3.0 & 1.7 & 44.6 & 53.6 & Silty Clay & Core $5-1$ & & & & & \\
\hline $14-4$ & 3.0 & 3.3 & 31.9 & 64.8 & Silty Clay & Core 5-1 & 8.0 & 15.0 & 32.1 & 53.0 & Silty Clay \\
\hline $14-5$ & 0.0 & 1.8 & 49.5 & 48.7 & Clayey Silt & $5-2$ & 4.0 & 18.3 & 38.7 & 43.0 & Silty Clay \\
\hline $14-6$ & 4.0 & 8.5 & 52.4 & 39.1 & Clayey Silt & $5-3$ & 8.0 & 14.1 & 37.0 & 48.9 & Silty Clay \\
\hline & & & & & & $5-4$ & 8.0 & 25.6 & 41.1 & 33.3 & Sand-Silt-Clay \\
\hline Hole 63.2 & & & & & & $5-5$ & 4.0 & 24.1 & 39.0 & 36.9 & Sand-Silt-Clay \\
\hline Core 1-1 & 2.0 & 5.9 & 31.8 & 62.3 & Silty Clay & $5-6$ & 5.0 & 16.2 & 43.5 & 40.3 & Clayey Silt \\
\hline $1-2$ & 1.0 & 14.6 & 42.0 & 43.4 & Silty Clay & & & & & & \\
\hline $1-3$ & 1.0 & 2.8 & 42.3 & 54.9 & Silty Clay & Core 6-1 & 4.0 & 12.0 & 51.9 & 36.1 & Clayey Silt \\
\hline $1-4$ & 5.0 & 5.3 & 30.6 & 64.1 & Silty Clay & $6-2$ & 3.0 & 9.4 & 60.2 & 30.4 & Clayey Silt \\
\hline Core 2-1 & 2.0 & 1.9 & 38.7 & 59.4 & Silty Clay & $6-3$ & 4.0 & 8.4 & 55.7 & 35.9 & Clayey Silt \\
\hline $2-2$ & 1.0 & 2.2 & 40.0 & 57.8 & Silty Clay & $6-4$ & 4.0 & 13.7 & 55.9 & 30.4 & Clayey Silt \\
\hline $2-3$ & 1.0 & 0.9 & 33.9 & 65.1 & Silty Clay & $6-5$ & 4.0 & 11.6 & 56.7 & 31.7 & Clayey Silt \\
\hline $2-4$ & 1.0 & 1.7 & 30.6 & 67.7 & Silty Clay & $6-6$ & 3.0 & 16.6 & 50.0 & 33.4 & Clayey Silt \\
\hline $2-6$ & 1.0 & 1.0 & 38.5 & 60.5 & Silty Clay & Core $7-1$ & 22.0 & 18.0 & 47.1 & 34.9 & Clayey Silt \\
\hline & & & & & & $7-2$ & 19.0 & 13.2 & 41.5 & 45.4 & Silty Clay \\
\hline Core $3-1$ & 39.0 & 1.3 & 38.1 & 60.5 & Silty Clay & $7-3$ & 6.0 & 13.5 & 40.1 & 46.4 & Silty Clay \\
\hline $3-2$ & 1.0 & 1.1 & 33.1 & 65.8 & Silty Clay & $7-4$ & 17.0 & 10.4 & 40.5 & 49.2 & Silty Clay \\
\hline $3-3$ & 1.0 & 3.3 & 22.9 & 73.9 & Silty Clay & $7-5$ & 11.0 & 13.0 & 43.7 & 43.2 & Clayey Silt \\
\hline $3-4$ & 2.0 & 2.3 & 25.1 & 72.6 & Silty Clay & $7-6$ & 7.0 & 16.8 & 45.1 & 38.1 & Clayey Silt \\
\hline Hole 64.0 & & & & & & Core $8-2$ & 2.0 & 7.5 & 33.9 & 58.6 & Silty Clay \\
\hline Core 1-1 & 7.0 & 35.7 & 20.8 & 43.5 & Sand-Silt-Clay & $8-3$ & 1.0 & 5.1 & 51.1 & 43.8 & Clayey Silt \\
\hline $1-2$ & 29.0 & 35.3 & 28.8 & 36.0 & Sand-Silt-Clay & & & & & & \\
\hline $1-3$ & 2.0 & 41.3 & 26.2 & 32.5 & Sand-Silt-Clay & Core $10-1$ & 25.0 & 6.9 & 49.8 & 43.3 & Clayey Silt \\
\hline $1-4$ & 3.0 & 32.8 & 25.0 & 42.2 & Sand-Silt-Clay & $10-2$ & 2.0 & 10.1 & 51.7 & 38.2 & Clayey Silt \\
\hline
\end{tabular}

${ }^{\mathrm{a}}$ Grainsize: Sand per cent of total weight greater than .062 millimeter; clay per cent of total weight less than .0039 millimeter; silt remainder of total weight. 
TABLE 1 - Continued

\begin{tabular}{|c|c|c|c|c|c|c|c|c|c|c|c|}
\hline \multicolumn{6}{|c|}{ Grainsize $^{\mathrm{a}}$} & \multicolumn{6}{|c|}{ Grainsize $^{\mathrm{a}}$} \\
\hline $\begin{array}{l}\text { Identifi- } \\
\text { cation }\end{array}$ & $\begin{array}{c}\text { Interval } \\
\mathrm{cm}\end{array}$ & $\begin{array}{c}\text { Sand } \\
\text { Per Cent }\end{array}$ & $\begin{array}{c}\text { Silt } \\
\text { Per Cent }\end{array}$ & $\begin{array}{c}\text { Clay } \\
\text { Per Cent }\end{array}$ & Cassification & $\begin{array}{l}\text { Identifi- } \\
\text { cation }\end{array}$ & $\begin{array}{c}\text { Interval } \\
\mathrm{cm}\end{array}$ & $\begin{array}{c}\text { Sand } \\
\text { Per Cent }\end{array}$ & $\begin{array}{c}\text { Silt } \\
\text { Per Cent }\end{array}$ & $\begin{array}{c}\text { Clay } \\
\text { Per Cent }\end{array}$ & Classification \\
\hline Hole 64.1 & & & & & & $9-2$ & 2.0 & 3.9 & 49.9 & 46.3 & Clayey Silt \\
\hline Core 1-1 & 19.0 & 19.1 & 44.7 & 36.2 & Clayey Silt & $9-3$ & 3.0 & 4.0 & 52.7 & 43.3 & Clayey Silt \\
\hline $1-2$ & 2.0 & 14.5 & 47.3 & 38.2 & Clayey Silt & Core $10-1$ & 12.0 & 1.4 & 53.3 & 45.3 & Clayey Silt \\
\hline $1-3$ & 5.0 & 14.4 & 45.7 & 39.9 & Clayey Silt & $10-2$ & 8.0 & 6.1 & 55.3 & 38.6 & Clayey Silt \\
\hline $1-4$ & 4.0 & 19.0 & 42.2 & 38.7 & Clayey Silt & Hole 65.0 & & & & & \\
\hline $1-5$ & 4.0 & 18.4 & 48.1 & 33.4 & Clayey Silt & & & & & & \\
\hline $1-6$ & 10.0 & 21.0 & 47.3 & 31.7 & Sand-Silt-Clay & Core $2-2$ & 2.0 & 11.0 & 29.7 & 59.3 & Silty Clay \\
\hline Core 2-1 & 25.0 & 22.6 & 42.5 & 34.9 & Sand-Silt-Clay & $2-3$ & 18.0 & 14.7 & 26.2 & 59.1 & Silty Clay \\
\hline $2-2$ & 6.0 & 23.1 & 47.6 & 29.3 & Sand-Silt-Clay & $2-4$ & 19.0 & 6.3 & 32.9 & 60.8 & Silty Clay \\
\hline $2-3$ & 20.0 & 21.6 & 42.8 & 35.6 & Sand-Silt-Clay & $2-5$ & 42.0 & 3.9 & 31.3 & 64.8 & Silty Clay \\
\hline $2-4$ & 3.0 & 20.1 & 47.9 & 32.0 & Sand-Silt-Clay & Core 3-1 & 15.0 & 2.5 & 32.1 & 65.3 & Silty Clay \\
\hline $2-5$ & 5.0 & 13.5 & 46.3 & 40.2 & Clayey Silt & $3-2$ & 31.0 & 12.4 & 31.0 & 56.6 & Silty Clay \\
\hline $2-6$ & 3.0 & 16.0 & 54.8 & 29.2 & Clayey Silt & $3-3$ & 2.0 & 4.2 & 32.7 & 63.1 & Silty Clay \\
\hline Core 3-1 & 9.0 & 23.5 & 51.7 & 24.7 & Sand-Silt-Clay & $3-4$ & 20.0 & 5.9 & 37.8 & 56.3 & Silty Clay \\
\hline $3-2$ & 3.0 & 10.9 & 61.7 & 27.5 & Clayey Silt & $3-5$ & 2.0 & 7.2 & 33.9 & 58.8 & Silty Clay \\
\hline $3-3$ & 7.0 & 11.5 & 59.5 & 29.0 & Clayey Silt & Core 4-2 & 12.0 & 13.9 & 30.5 & 55.6 & Silty Clay \\
\hline $3-4$ & 20.0 & 12.3 & 59.0 & 28.7 & Clayey Silt & $4-4$ & 4.0 & 10.7 & 36.1 & 53.2 & Silty Clay \\
\hline $3-5$ & 17.0 & 8.5 & 57.7 & 33.8 & Clayey Silt & $4-5$ & 4.0 & 5.0 & 28.4 & 66.5 & Silty Clay \\
\hline $3-6$ & 14.0 & 9.1 & 55.5 & 35.5 & Clayey Silt & Core 5-2 & 38.0 & 12.0 & 36.4 & 51.5 & Silty Clay \\
\hline Core 4-1 & 10.0 & 16.9 & 48.8 & 34.3 & Clayey Silt & $5-4$ & 59.0 & 11.6 & 38.4 & 50.0 & Silty Clay \\
\hline $4-2$ & 3.0 & 13.2 & 54.0 & 32.8 & Clayey Silt & $5-5$ & 1.0 & 11.8 & 38.8 & 49.4 & Silty Clay \\
\hline $4-3$ & 4.0 & 16.2 & 49.8 & 34.0 & Clayey Silt & $5-6$ & 21.0 & 11.5 & 39.3 & 49.3 & Silty Clay \\
\hline $4-4$ & 3.0 & 10.1 & 53.6 & 36.3 & Clayey Silt & Core 6-2 & 40.0 & 16.1 & 35.2 & 48.7 & Silty Clay \\
\hline $4-5$ & 4.0 & 9.9 & 49.4 & 40.7 & Clayey Silt & Core $7-2$ & 82.0 & 15.4 & 34.4 & 50.1 & Silty Clay \\
\hline $4-6$ & 2.0 & 9.1 & 54.7 & 36.2 & Clayey Silt & 7-3 & 7.0 & 10.6 & 35.8 & 53.6 & Silty Clay \\
\hline Core $5-1$ & 25.0 & 8.1 & 55.9 & 36.1 & Clayey Silt & $7-4$ & 29.0 & 13.1 & 36.8 & 50.1 & Silty Clay \\
\hline $5-2$ & 97.0 & 6.7 & 55.0 & 38.3 & Clayey Silt & $7-5$ & 16.0 & 18.4 & 32.2 & 49.4 & Silty Clay \\
\hline $5-3$ & 4.0 & 10.4 & 56.0 & 33.6 & Clayey Silt & Core $8-1$ & 3.0 & 21.8 & 34.3 & 43.9 & Sand-Silt-Clay \\
\hline $5-4$ & 2.0 & 11.8 & 62.2 & 26.1 & Clayey Silt & $8-2$ & 53.0 & 23.5 & 32.3 & 44.1 & Sand-Silt-Clay \\
\hline $5-5$ & 2.0 & 11.2 & 58.4 & 30.4 & Clayey Silt & $8-3$ & 66.0 & 39.5 & 27.6 & 32.9 & Sand-Silt-Clay \\
\hline $3-6$ & 1.0 & 12.5 & 54.3 & 33.2 & Clayey silt & $8-4$ & 2.0 & 18.3 & 36.5 & 45.2 & Silty Clay \\
\hline Core $6-1$ & 3.0 & 19.2 & 49.7 & 31.1 & Clayey Silt & $8-5$ & 2.0 & 22.1 & 38.5 & 39.4 & Sand-Silt-Clay \\
\hline $6-2$ & 2.0 & 15.8 & 51.1 & 33.1 & Clayey Silt & $8-6$ & 2.0 & 28.2 & 32.7 & 39.1 & Sand-Silt-Clay \\
\hline $6-3$ & 2.0 & 19.0 & 51.5 & 29.6 & Clayey Silt & Core 9-1 & 21.0 & 10.7 & 41.7 & 47.5 & Silty Clay \\
\hline $6-4$ & 4.0 & 17.1 & 44.8 & 38.1 & Clayey Silt & 9-2 & 2.0 & 16.9 & 32.7 & 50.5 & Silty Clay \\
\hline Core 7-1 & 99.0 & 9.9 & 55.6 & 34.5 & Clayey Silt & $9-3$ & 2.0 & 29.0 & 33.3 & 37.6 & Sand-Silt-Clay \\
\hline $7-2$ & 4.0 & 6.9 & 49.2 & 43.9 & Clayey Silt & $9-4$ & 2.0 & 33.3 & 29.9 & 36.7 & Sand-Silt-Clay \\
\hline $7-3$ & 5.0 & 8.3 & 52.1 & 39.6 & Clayey Silt & $9-5$ & 88.0 & 12.2 & 39.8 & 48.0 & Silty Clay \\
\hline $7-4$ & 4.0 & 5.1 & 54.1 & 40.8 & Clayey Silt & $9-6$ & 10.0 & 14.2 & 41.3 & 44.5 & Silty Clay \\
\hline Core $8-1$ & 100.00 & 7.4 & 51.2 & 41.4 & Clayey Silt & Core 10-1 & 70.0 & 12.3 & 41.2 & 46.5 & Silty Clay \\
\hline $8-2$ & 1.0 & 5.4 & 50.5 & 44.1 & Clayey Silt & $10-2$ & 2.0 & 21.8 & 37.0 & 41.3 & Sand-Silt-Clay \\
\hline Core 9-1 & 9.0 & 4.7 & 45.7 & 49.5 & Silty Clay & $10-3$ & 53.0 & 26.7 & 31.0 & 42.3 & Sand-Silt-Clay \\
\hline
\end{tabular}

${ }^{a}$ Grainsize: Sand per cent of total weight greater than .062 millimeter; clay per cent of total weight less than .0039 millimeter; silt remainder of total weight. 
TABLE 1 - Continued

\begin{tabular}{|c|c|c|c|c|c|c|c|c|c|c|c|}
\hline \multirow[b]{2}{*}{$\begin{array}{l}\text { Identifi- } \\
\text { cation }\end{array}$} & \multirow[b]{2}{*}{$\begin{array}{c}\text { Interval } \\
\mathrm{cm}\end{array}$} & \multicolumn{3}{|c|}{ Grainsize $^{a}$} & \multirow[b]{2}{*}{ Classification } & \multirow[b]{2}{*}{$\begin{array}{l}\text { Identifi- } \\
\text { cation }\end{array}$} & \multicolumn{5}{|c|}{ Grainsize $^{\mathrm{a}}$} \\
\hline & & $\begin{array}{c}\text { Sand } \\
\text { Per Cent }\end{array}$ & $\begin{array}{c}\text { Silt } \\
\text { Per Cent }\end{array}$ & $\begin{array}{c}\text { Clay } \\
\text { Per Cent }\end{array}$ & & & $\begin{array}{c}\text { Interval } \\
\mathrm{cm}\end{array}$ & $\begin{array}{c}\text { Sand } \\
\text { Per Cent }\end{array}$ & $\begin{array}{c}\text { Silt } \\
\text { Per Cent }\end{array}$ & $\begin{array}{c}\text { Clay } \\
\text { Per Cent }\end{array}$ & Classification \\
\hline $10-4$ & 2.0 & 22.2 & 35.2 & 42.7 & Sand-Silt-Clay & Hole 66.0 & & & & & \\
\hline Core $11-2$ & 65.0 & 27.3 & 34.2 & 38.6 & Sand-Silt-Clay & Core 2-1 & 2.0 & 7.8 & 38.0 & 54.2 & Silty Clay \\
\hline $11-3$ & 2.0 & 4.6 & 50.9 & 44.5 & Clayey Silt & $2-2$ & 9.0 & 14.3 & 38.6 & 47.0 & Silty Clay \\
\hline $11-4$ & 51.0 & 17.9 & 43.9 & 38.1 & Clayey Silt & $2-3$ & 4.0 & 14.5 & 42.7 & 42.8 & Silty Clay \\
\hline $11-5$ & 41.0 & 16.8 & 39.2 & 44.0 & Silty Clay & Core 3-1 & 20.0 & 16.4 & 40.9 & 42.7 & Silty Clay \\
\hline $11-6$ & 2.0 & 18.5 & 45.5 & 36.0 & Clayey Silt & $3-2$ & 4.0 & 23.9 & 38.3 & 37.8 & Sand-Silt-Clay \\
\hline Core $12-1$ & 4.0 & 30.4 & 39.2 & 30.4 & Sand-Silt-Clay & $3-3$ & 3.0 & 21.7 & 39.9 & 38.4 & Sand-Silt-Clay \\
\hline $12-2$ & 25.0 & 26.8 & 43.4 & 29.8 & Sand-Silt-Clay & $3-4$ & 12.0 & 20.1 & 40.3 & 39.6 & Sand-Silt-Clay \\
\hline $12-3$ & 3.0 & 8.4 & 16.9 & 74.7 & Silty Clay & $3-5$ & 17.0 & 29.9 & 35.6 & 34.4 & Sand-Silt-Clay \\
\hline $12-4$ & 36.0 & 31.3 & 30.8 & 37.9 & Sand-Silt-Clay & $3-6$ & 3.0 & 26.6 & 39.5 & 33.9 & Sand-Silt-Clay \\
\hline $12-5$ & 1.0 & 31.2 & 36.4 & 32.5 & Sand-Silt-Clay & Core 6-1 & 6.0 & 0.0 & 11.8 & 88.2 & Clay \\
\hline $12-6$ & 8.0 & 38.3 & 37.9 & 23.8 & Sand-Silt-Clay & $6-2$ & 2.0 & 0.0 & 8.5 & 91.5 & Clay \\
\hline Core 13-1 & 30.0 & 45.5 & 34.9 & 19.6 & Silty Sand & $6-3$ & 2.0 & 0.0 & 7.4 & 92.6 & Clay \\
\hline $13-2$ & 14.0 & 30.3 & 41.1 & 28.6 & Sand-Silt-Clay & $6-4$ & 2.0 & 0.0 & 25.7 & 74.3 & Silty Clay \\
\hline $13-3 c$ & 2.0 & 31.0 & 42.0 & 27.0 & Sand-Silt-Clay & Core $7-1$ & 30.0 & 0.0 & 12.3 & 87.7 & Clay \\
\hline $13-4$ & 2.0 & 43.8 & 36.3 & 19.9 & Silty Sand & $7-2$ & 2.0 & 0.0 & 12.5 & 87.5 & Clay \\
\hline $13-5$ & 2.0 & 41.5 & 32.8 & 25.7 & Sand-Silt-Clay & $7-3$ & 2.0 & 0.0 & 12.8 & 87.2 & Clay \\
\hline $13-6$ & 9.0 & 38.3 & 40.0 & 21.7 & Sand-Silt-Clay & $7-4$ & 2.0 & 0.0 & 11.9 & 88.1 & Clay \\
\hline Core 14-1 & 3.0 & 29.9 & 40.0 & 30.1 & Sand-Silt-Clay & Core 8-1 & 9.0 & 0.0 & 9.1 & 90.9 & Clay \\
\hline $14-2$ & 2.0 & 43.9 & 33.0 & 23.1 & Sand-Silt-Clay & $8-2$ & 10.0 & 0.0 & 42.9 & 57.1 & Silty Clay \\
\hline $14-3$ & 2.0 & 52.3 & 29.6 & 18.1 & Silty Sand & $8-3$ & 3.0 & 0.0 & 9.8 & 90.2 & Clay \\
\hline $14-4$ & 14.0 & 28.8 & 39.6 & 31.5 & Sand-Silt-Clay & $8-4$ & 12.0 & 0.0 & 11.6 & 88.4 & Clay \\
\hline $14-5$ & 1.0 & 30.0 & 37.9 & 32.1 & Sand-Silt-Clay & $8-5$ & 23.0 & 0.0 & 9.1 & 90.9 & Clay \\
\hline $14-6$ & 2.0 & 29.2 & 32.8 & 38.0 & Sand-Silt-Clay & Core 9-1 & 117.0 & 0.0 & 27.7 & 72.3 & Silty Clay \\
\hline Core $16-2$ & 43.0 & 36.5 & 34.6 & 28.9 & Sand-Silt-Clay & Hole 66.1 & & & & & \\
\hline $16-3$ & 3.0 & 40.6 & 31.9 & 27.5 & Sand-Silt-Clay & & & & & & \\
\hline $16-4$ & 2.0 & 35.3 & 33.6 & 31.1 & Sand-Silt-Clay & Core 2-1 & 8.0 & 0.6 & 33.2 & 66.2 & Silty Clay \\
\hline $16-5$ & 2.0 & 31.7 & 36.3 & 32.0 & Sand-Silt-Clay & $2-2$ & 49.0 & 0.8 & 20.4 & 78.8 & Clay \\
\hline $16-6$ & 3.0 & 37.2 & 28.9 & 33.9 & Sand-Silt-Clay & $2-3$ & 6.0 & 1.3 & 28.4 & 70.3 & Silty Clay \\
\hline Hole 65.1 & & & & & & $2-4$ & 12.0 & 1.7 & 30.8 & 67.5 & Silty Clay \\
\hline Core 2-1 & 970 & 11 & 50.7 & $48 ?$ & Cla & $2-5$ & 2.0 & 1.5 & 34.1 & 64.4 & Silty Clay \\
\hline & & & 50.1 & 40.2 & Clay & $2-6$ & 2.0 & 1.1 & 31.5 & 67.4 & Silty Clay \\
\hline Core 4-1 & 33.0 & 28.9 & 39.2 & 32.0 & Sand-Silt-Clay & Core $3-1$ & 33.0 & 5.3 & 31.0 & 63.7 & Silty Clay \\
\hline $4-2$ & 2.0 & 27.8 & 41.0 & 31.2 & Sand-Silt-Clay & $3-2$ & 2.0 & 11.4 & 30.6 & 57.9 & Silty Clay \\
\hline $4-3$ & 2.0 & 32.3 & 37.0 & 30.7 & Sand-Silt-Clay & $3-3$ & 2.0 & 11.6 & 27.6 & 60.8 & Silty Clay \\
\hline $4-4$ & 1.0 & 28.5 & 41.5 & 30.1 & Sand-Silt-Clay & $3-4$ & 2.0 & 7.7 & 35.6 & 56.8 & Silty Clay \\
\hline $4-5$ & 13.0 & 21.9 & 46.0 & 32.2 & Sand-Silt-Clay & $3-5$ & 2.0 & 4.8 & 34.6 & 60.6 & Silty Clay \\
\hline $4-6$ & 1.0 & 7.3 & 44.3 & 48.3 & Silty Clay & $3-6$ & 2.0 & 5.5 & 30.3 & 64.2 & Silty Clay \\
\hline Core $5-1$ & 57.0 & 23.1 & 47.0 & 29.9 & Sand-Silt-Clay & Core 4-1 & 101.0 & 3.5 & 30.8 & 65.7 & Silty Clay \\
\hline $5-2$ & 3.0 & 23.2 & 42.7 & 34.1 & Sand-Silt-Clay & $4-2$ & 2.0 & 3.8 & 26.7 & 69.5 & Silty Clay \\
\hline $5-3$ & 2.0 & 26.7 & 41.3 & 32.0 & Sand-Silt-Clay & $4-3$ & 30.0 & 5.2 & 21.1 & 73.7 & Silty Clay \\
\hline $5-4$ & 3.0 & 32.0 & 37.5 & 30.5 & Sand-Silt-Clay & $4-4$ & 5.0 & 15.7 & 35.3 & 49.0 & Silty Clay \\
\hline
\end{tabular}

\footnotetext{
${ }^{\mathrm{a}}$ Grainsize: Sand per cent of total weight greater than .062 millimeter; clay per cent of total weight less than .0039 millimeter; silt
} remainder of total weight. 
TABLE 1 - Continued

\begin{tabular}{|c|c|c|c|c|c|}
\hline \multirow[b]{2}{*}{$\begin{array}{l}\text { Identifi- } \\
\text { cation }\end{array}$} & \multicolumn{5}{|c|}{ Grainsize $^{a}$} \\
\hline & $\begin{array}{c}\text { Interval } \\
\mathrm{cm}\end{array}$ & $\begin{array}{c}\text { Sand } \\
\text { Per Cent }\end{array}$ & $\begin{array}{c}\text { Silt } \\
\text { Per Cent }\end{array}$ & $\begin{array}{c}\text { Clay } \\
\text { Per Cent }\end{array}$ & Classification \\
\hline \multicolumn{6}{|c|}{ Hole 66.1 - Continued } \\
\hline $4-5$ & 27.0 & 9.9 & 35.4 & 54.7 & Silty Clay \\
\hline $4-6$ & 50.0 & 10.8 & 40.5 & 48.7 & Silty Clay \\
\hline Core 5-1 & 3.0 & 16.0 & 34.6 & 49.4 & Silty Clay \\
\hline $5-2$ & 3.0 & 8.7 & 40.2 & 51.1 & Silty Clay \\
\hline $5-3$ & 18.0 & 7.5 & 39.9 & 52.6 & Silty Clay \\
\hline $5-4$ & 11.0 & 11.0 & 36.1 & 52.9 & Silty Clay \\
\hline $5-5$ & 19.0 & 18.9 & 36.0 & 45.1 & Silty Clay \\
\hline $5-6$ & 20.0 & 11.6 & 36.1 & 52.3 & Silty Clay \\
\hline Core 6-2 & 12.0 & 7.8 & 27.4 & 64.8 & Silty Clay \\
\hline $6-3$ & 23.0 & 8.1 & 30.2 & 61.6 & Silty Clay \\
\hline $6-4$ & 32.0 & 10.5 & 35.0 & 54.5 & Silty Clay \\
\hline $6-5$ & 18.0 & 15.4 & 37.0 & 47.6 & Silty Clay \\
\hline $6-6$ & 16.0 & 15.2 & 33.7 & 51.1 & Silty Clay \\
\hline Core $7-1$ & 11.0 & 10.1 & 40.0 & 49.9 & Silty Clay \\
\hline $7-2$ & 20.0 & 14.3 & 41.4 & 44.3 & Silty Clay \\
\hline $7-3$ & 102.0 & 13.0 & 38.1 & 48.9 & Silty Clay \\
\hline $7-4$ & 3.0 & 10.0 & 40.9 & 49.1 & Silty Clay \\
\hline $7-5$ & 14.0 & 9.1 & 0.2 & 50.7 & Silty Clay \\
\hline $7-6$ & 7.0 & 9.9 & 41.1 & 49.0 & Silty Clay \\
\hline Core 8-1 & 2.0 & 8.5 & 38.2 & 53.2 & Silty Clay \\
\hline $8-2$ & 11.0 & 10.3 & 40.1 & 49.6 & Silty Clay \\
\hline $8-3$ & 11.0 & 13.7 & 39.9 & 46.3 & Silty Clay \\
\hline $8-5$ & 13.0 & 14.0 & 41.6 & 44.4 & Silty Clay \\
\hline $8-6$ & 13.0 & 14.0 & 41.6 & 44.4 & Silty Clay \\
\hline \multicolumn{6}{|l|}{ Hole 67.0} \\
\hline Core 1-1 & 27.0 & 5.8 & 61.2 & 33.0 & Clayey Silt \\
\hline \multicolumn{6}{|l|}{ Hole 67.1} \\
\hline Core $1-1$ & 61.0 & 0.2 & 55.2 & 44.5 & Clayey Silt \\
\hline
\end{tabular}

${ }^{a}$ Grainsize: Sand per cent of total weight greater than .062 millimeter; clay per cent of total weight less than .0039 millimeter; silt remainder of total weight. 